\title{
MODEL OF QUBIT IN MULTI-ELECTRON QUANTUM DOT
}

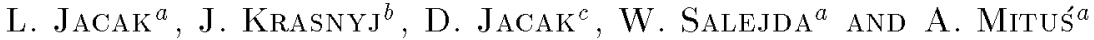 \\ ${ }^{a}$ Institute of Physics, Wrocław University of Technology \\ Wybrzeże Wyspiańskiego 27, 50-370 Wrocław, Poland \\ ${ }^{b}$ Institute of Mathematics, University of Opole, Oleska 48, Opole, Poland \\ (on leave from Institute of Physics, University of Odessa, Odessa, Ukraine) \\ ${ }^{c}$ Institute of Mathematics, Wrocław University of Technology \\ Wybrzeże Wyspiańskiego 27, 50-370 Wrocław, Poland
}

(Received August 25, 2000; in final form October 27, 2000)

\begin{abstract}
The discussion of qubit for quantum computation in quantum dots technology is presented. The state-of-the-art structure of multi-electron dot is considered and the appropriate quasi-two-level system is suggested employing the singlet-triplet transition in the presence of magnetic field. The methods of qubit rotation (the write procedure) as well as two-qubit operations, as controlled-NOT, in vertically stacked dots system are analysed.
\end{abstract}

PACS numbers: 73.20.Dx

\section{Introduction}

Recently, several proposals for the quantum information processing on quantum dots (QDs) systems have been suggested. They include both the spin degree of freedom [1,2], as well as the carrier density excitations with special attention paid to excitons in self-assembled dots $[3,4]$. The latter idea seems to be interesting, since it employs the ultrafast resonant optical techniques [5,6], which are faster than typical decoherence processes involving electrons and holes in dots. On the other hand, the advantage of the spin degrees of freedom is based on the fact that the decoherence for spin is less efficient [2, 7]. All the proposals refer however to ideal situations of qubit definition as the exactly two-level system [8, 9], e.g. spin of single electron or exactly one exciton per dot, whereas in real QDs we deal rather with multi-electron systems with complicated level structure. In particular it concerns the self-assembled strain-induced vertically stacked dots pair, interesting for applications $[10,11]$. Therefore the proper definition of qubits in such structures needs the discussion. For instance, instead of single electron spin, the noncompensated total spin of electrons could be used as a qubit. In practice, an isolated from 
environment subsystem could play the role of a qubit if two quantum levels of this subsystem are sufficiently well separated from the rest of its spectrum.

As it follows from a theoretical analysis of filling many-electron dots, and even from recent experiments on vertical dots [12-14], a common and qualitative property of multi-electron dots is the existence of the so-called singlet-triplet transitions between the effective single-particle levels in magnetic field (for a number of electrons $N \geq 4$ ). It is a result of the competition between the direct Coulomb interaction and the exchange interaction of electrons in a nonzero perpendicular magnetic field in the region of crossing single-particle (Fock-Darwin) levels (cf. e.g. [15]). The magnetic field changes the energy separations of these levels, and one can observe the departure from antiferromagnetic spin alignment to ferromagnetic filling according to first Hund's rule, similar to ordinary atoms. The characteristic crossing of levels with the simultaneous change of the spin polarization seems to be the dominant qualitative picture within the shell description of the dot, robust against many particularities of theoretical modelling [15, 16], and is convincingly confirmed in experiment [12] in an easily attainable region of magnetic field (of the order of $1 \mathrm{~T}$ ). Two crossing levels, well-separated from others, could be considered as a qubit in the realistic multi-electron dot. The energy distance between these levels can be precisely tuned by the magnetic field and can be made very small, which is important as the Zeeman splitting is also minute. A too small energy distance between qubit levels is however inconvenient, as it leads to the long time of qubit rotation (e.g. for $\sim 1 \mu \mathrm{eV}$ of the order of $10^{-10} \mathrm{~s}$ ). We analyse the possibility of proper definition of the qubit employing the singlet-triplet transition for multi-electron dot (with $N \geq 4$ ) and also singlet-triplet transition in the model He-dot, i.e. two-electron system (cf. $[16,17]$ ). The methods of qubit rotations are considered. The two-qubit system is modelled by a pair of vertically stacked dots, each with two electrons, interacting electrically.

\section{Properties of state-of-the-art self-assembled QDs}

The electrical interaction in QDs plays a much more important role than in ordinary atoms. The quantization of kinetic energy by confining potential scales with QD dimension $d$ as $1 / d^{2}$, while the Coulomb interaction energy as $1 / d$. For small dots $(d \sim 10-20 \mathrm{~nm})$ these two terms are in the similar relation as in atoms and Hund's rules are applied for noncompletely filled electrons shells. According to these rules the total spin $S$ within the shell is maximal and the same holds for the total angular momentum. By varying the external magnetic field perpendicular to the dot it is possible to change the effective dot radius $d$, because the magnetic field enhances confinement. It results in variation of inter-level energy distances and specific interplay between direct and exchange interaction terms. It causes the transitions between levels, completely unaccessible for atoms or molecules. In order to induce similar behaviour in atoms the one-order greater magnetic fields should be applied, i.e. the fields of magnitude of a few tens or hundreds of $\mathrm{T}$ instead of single $\mathrm{T}$ as for $\mathrm{QD}$. This rare opportunity meets recently with growing interest in implementation of quantum logic gates in QDs technology. Several proposals are suggested, but for considerations of logic gating the idealized structures are 
assumed (as a single electron dot for a spin qubit, or a similar ideal structure for an excitonic qubit). Therefore the realistic considerations are of crucial importance (cf. e.g. [18-20] for realistic studies of excitons) especially addressed to coupled dots systems.

For the state-of-the-art coupled QDs systems we deal with two almost planar, lens-shaped vertically stacked dots $[10,11]$. They are self-assembled InAs/GaAs dots induced by the strain, in which several electrons (and holes) can be trapped. They have a radius of the order of $10 \mathrm{~nm}$ (the upper dot has however the slightly greater radius than the bottom one [10]). The vertical separation between dots in the pair is also of the order of $10 \mathrm{~nm}$.

The single self-assembled dot of similar dimension as each in this pair is very well recognized from the point of view of its electronic structure [15]. Let us remind the most important one of these properties.

I. The real geometry of confinement only weakly modifies the single-electron levels (and their dependence on magnetic field) from their form given by FockDarwin states (i.e. for planar parabolic confinement), cf. Fig. 1, where the comparison of pure $2 \mathrm{D}$ parabolic confinement with lens shape $3 \mathrm{D}$ confinement is presented (the lens radius $d=18 \mathrm{~nm}$ and the lens hight $h=4.4 \mathrm{~nm}$, cf. [21]). For this structure, the shells are clearly defined.

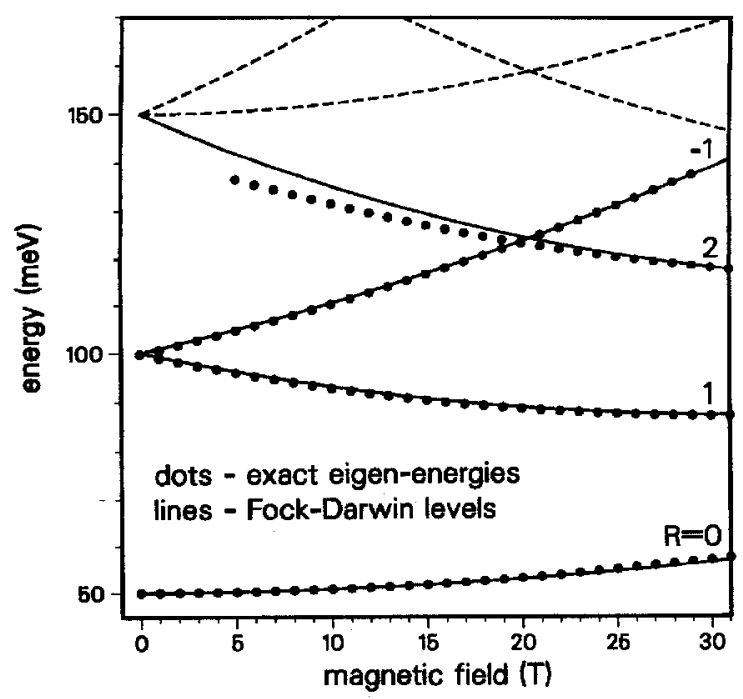

Fig. 1. Comparison of single-electron levels for lens-shaped self-assembled dot (radius $d=18 \mathrm{~nm}$, hight $h=4.4 \mathrm{~nm}$ ) of exact calculation (circles) and Fock-Darwin levels (lines) $[15,30]$; and Fock-Darwin levels not confined for lens-shaped dot (dashed lines).

II. Inclusion of interaction modifies single-particle levels especially strongly within shells. Generally, levels (shells) are shifted towards higher energies proportionally to the inter-particle interaction energy and a number of electrons (a typical interaction energy scale in the considered structure $\sim 25 \mathrm{meV}$ ). 
III. The magnetic field dependent interplay between direct Coulomb interaction and exchange interaction results in singlet-triplet transitions in a many-particle ground state for $N=4,5,6, \ldots$ ( $N$ - the number of electrons in the dot), cf. Fig. 2a. It can be also observed via the analysis of addition energy for the last electron (or chemical potential), cf. Fig. 2 b.
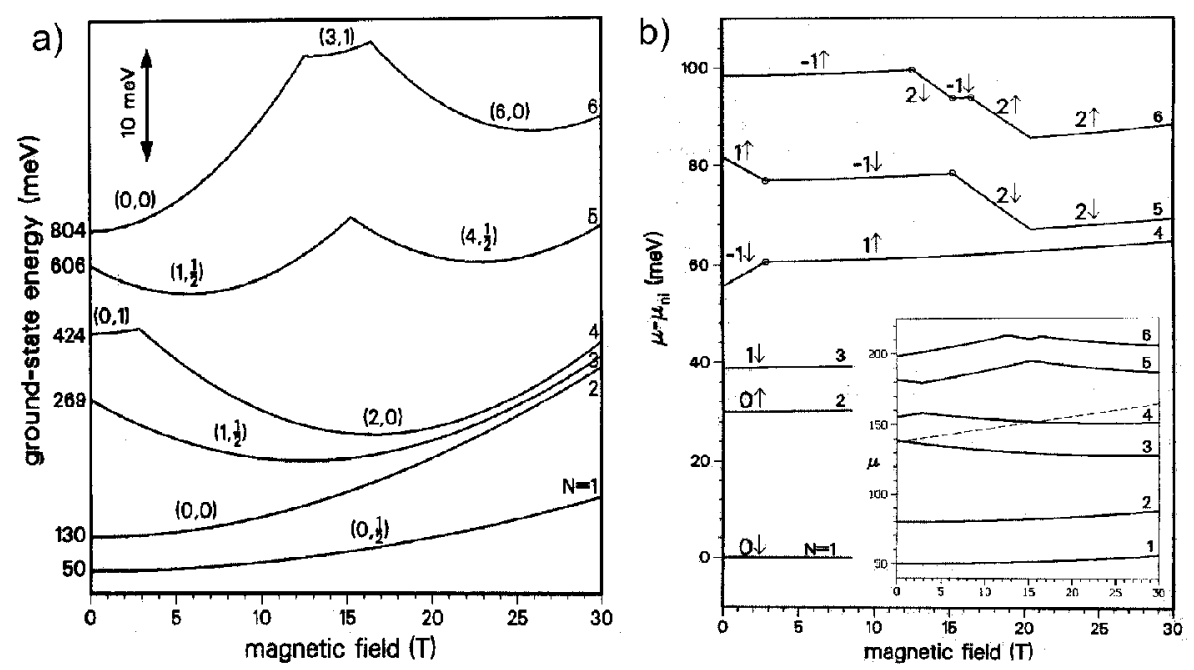

Fig. 2. The ground-state energies for confined $(N=1 \div 6)$ electrons in self-assembled QD $(d=18 \mathrm{~nm}, h=4.4 \mathrm{~nm})$; pairs of numbers on the curves $\left(L, S_{z}\right)$ (exact diagonalization) $[15,26]$ (a); difference between chemical potential of the interacting and noninteracting systems and chemical potential (inset) vs. magnetic field, angular momenta and spins of the single-particle states, to which electrons are added, are indicated (exact diagonalization) $[15,26]$ (b).

IV. The hole structure is similar to the electron one as the strain separates the subbands of light and heavy holes and therefore reduces their mixing, but different parameters result: $\hbar \omega_{0}^{\mathrm{h}} \sim 15 \mathrm{meV}$ (for electrons $\hbar \omega_{0}^{\mathrm{e}} \sim 30 \mathrm{meV}$ ) leading however to a similar number of confined shells as for electrons.

$\mathrm{V}$. For the fields of the order of $20-30 \mathrm{~T}$ ( at $\hbar \omega_{0}^{\mathrm{e}} \sim 30-50 \mathrm{meV}$ ) the transition between singlet-ground and triplet-excited states for $N=2$ can also be observed (note however that if $\hbar \omega_{0}^{e} \sim 5 \mathrm{meV}$ then this transition takes place at $\sim 3 \mathrm{~T}$, [17]).

VI. Proximity effects in a pair of the dots change significantly the level structure in both dots [22].

VII. The weakly interacting excitons can be created in the self-assembled dot, due to strong confinement both for electrons and holes and electron-hole attraction $\left(\hbar \omega_{0}^{\mathrm{e}}+\hbar \omega_{0}^{\mathrm{h}} \sim 45 \mathrm{meV}\right.$, and Coulomb energy $\left.\sim 25 \mathrm{meV}\right)$, cf. [15, 19, 20, 23, 24]. However, for the energy of electron excitations of the order of $30-50 \mathrm{meV}$, as for the considered dot, the strong multi-phonons mixing effects lead to the creation of polarons (with an energy shift of the order of several meV) [25], which strongly enhances decoherence for excitons via phonon channel. 


\section{Qubit definition, single-qubit rotations, two-qubit operations}

Let us focus the attention on the singlet-triplet transition for QDs. There are two types of this transition: the transition in multi-electron dot for $N \geq 4$ accompanying the Fock-Darwin levels intersection and the transition in the case of He dot, i.e. for $N=2$. The first type transition has been recently observed by Tarucha et al. $[12,13]$ for vertical, of medium size dot, cf. Fig. 3a. Let us note that the experimentally observed behaviour (Fig. 3a) very well corresponds with theoretical predictions (Fig. 3c) for a similar number of electrons, though for a distinct dot diameter.
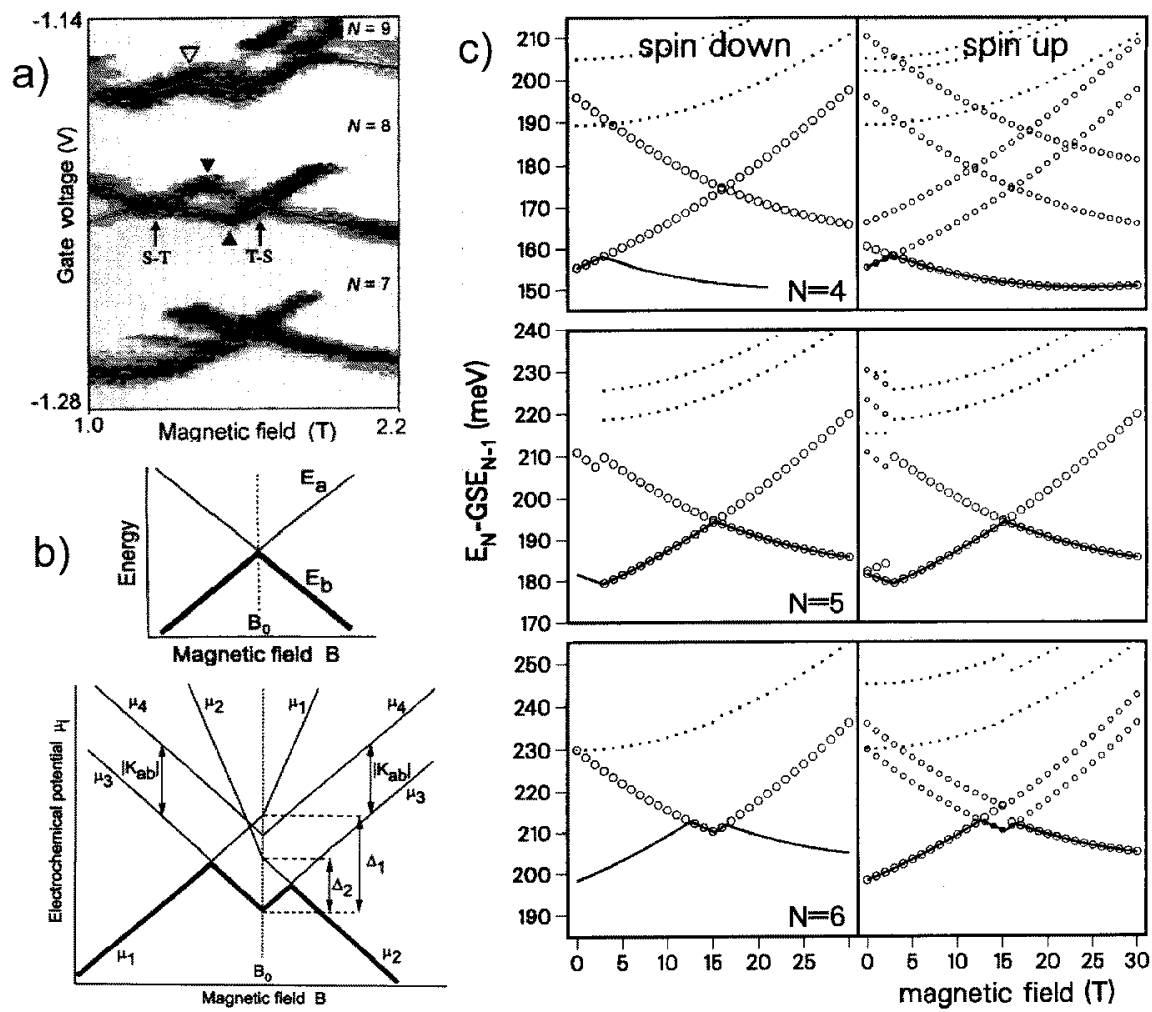

Fig. 3. The singlet-triplet transition $(N \geq 4)$ observed experimentally by Tarucha et al. in dot with $\hbar \omega_{0}^{\mathrm{e}} \sim 2 \mathrm{meV}$ (after [12], with Authors' permission) (a); the simplified two-electron model for singlet-triplet intersecting levels for $N \geq 4$ (after [12]) (b); addition energy spectrum vs. magnetic field calculated for self-assembled dot with $\hbar \omega_{0}^{\mathrm{e}} \sim$ $30 \mathrm{meV}$, areas of circles are proportional to the intensities of individual transitions; continuous lines - chemical potential $[15,26]$ (c).

Tarucha et al. gave also a skillful explanation of the many-particle system transition in terms of only the last pair of electrons [12] in the case when two various Fock-Darwin levels intersect (cf. Fig. 3b). Within this simple approach 
Tarucha et al. reproduce the same properties of chemical potential as found by exact diagonalization methods applied up to 6 electrons [26], cf. Fig. 2, as well as by the application of simplified Hubbard-type calculus [27].

The singlet-triplet transition for $N=2$ does not accompany the crossing of Fock-Darwin levels. It indicates rather the enhancement with magnetic field of the exchange energy which is inconvenient for the singlet state and favours the originally excited state - the triplet one - cf. Fig. 4. This type of transition appears at a sufficiently low field for dots with a not too strong confinement parameter, as the critical field $B^{*}[\mathrm{~T}] \sim \hbar \omega_{0}[\mathrm{meV}] / 1.6$. Therefore for a vertically stacked pair of self-assembled dots (with $\hbar \omega_{0}^{\mathrm{e}} \sim 30-50 \mathrm{meV}$ ) the transition field is in an uninteresting region (of the order of $20-30 \mathrm{~T}$ ), and for these dots rather the previous type of singlet-triplet transitions for $N \geq 4$ is attainable at lower fields - as it is indicated in Fig. 2.

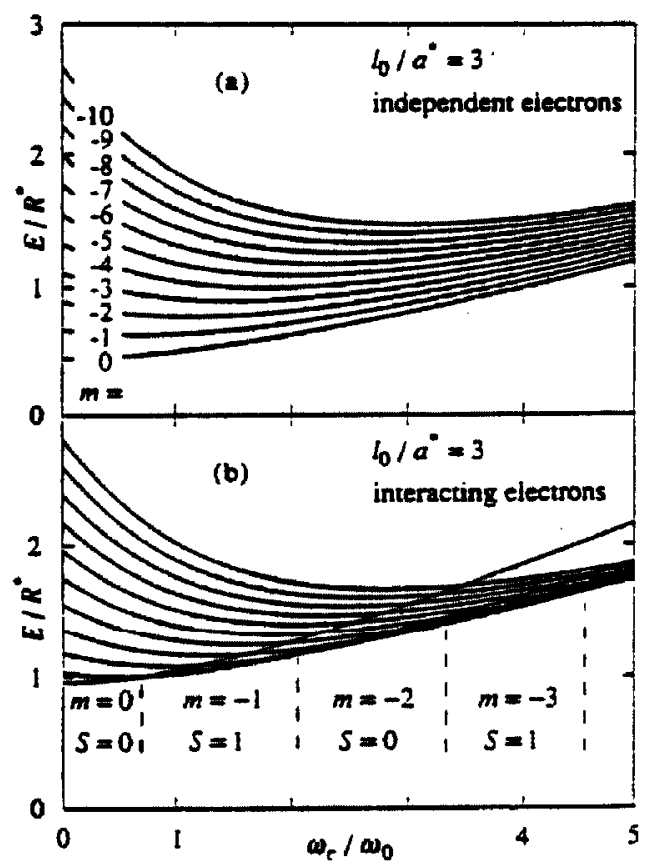

Fig. 4. The intersections of single and triple states for He QD, (a) independent electrons, (b) interacting electrons (after [17], with Authors' permission).

In the vicinity of the transition point, the two crossing levels lay very close. As the internal shells are completely filled and energetically distant from considered intersecting levels, the last two electrons create the almost separated subsystem (cf. Figs. 3 and 2). The intersecting two levels for the last electrons (e.g. in the Tarucha model) could be treated as a qubit (similarly as the singlet and triplet states for $N=2$ near the critical magnetic field, even though in this case we do not deal with the intersection of bare single-particle levels). Note that the Zeeman 


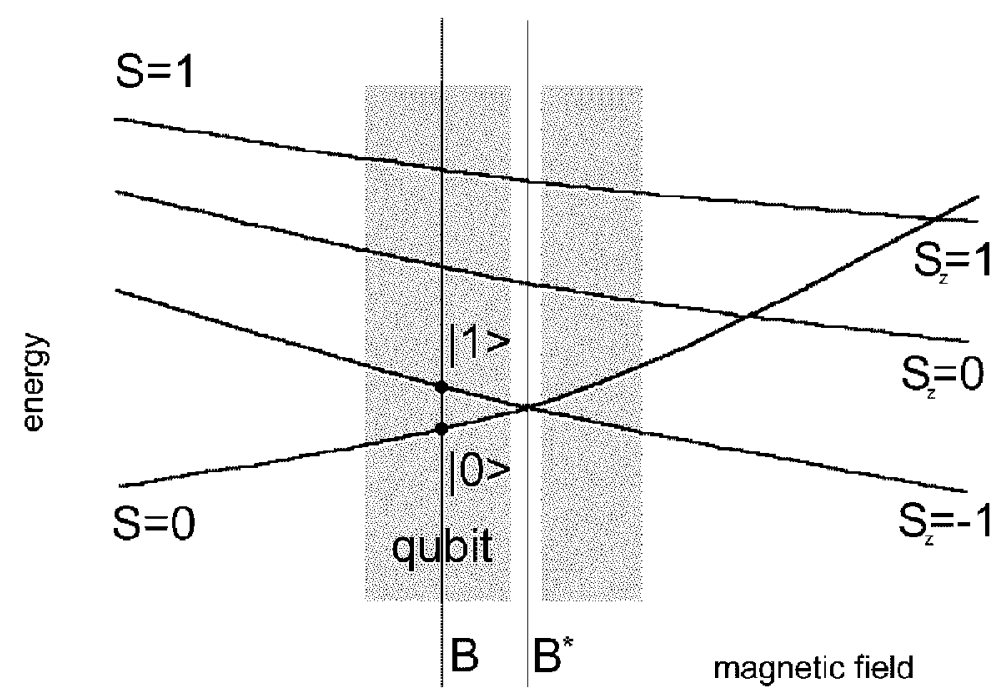

Fig. 5. The model of a qubit in the vicinity of the singlet-triplet transition point (scheme for He dot), the range of proper definition of the qubit is indicated on both sides of the critical field.

splitting for $\mathrm{GaAs}$ is $\sim 0.03 \mathrm{meV} / \mathrm{T}$ and it attains the value of $0.1 \mathrm{meV}$ at $3 \mathrm{~T}$. Hence the proper definition of the qubit needs the level separation to be smaller than the Zeeman splitting — what is attainable in the vicinity of critical field, cf. Fig. 5.

For a further model analysis we consider the singlet-triplet transition for $N=2$ assuming $\hbar \omega_{0}^{\mathrm{e}} \sim 5 \mathrm{meV}$. In the case of He dot the wave functions for ground and first excited states can be approximated by the analytical formulae given in Appendix A.

The excited triplet state, for the field smaller than the transition field, is long living owing to spin symmetry of the wave function and corresponding selection rules. We have analysed the transitions from this state to the ground state induced by various perturbations coupling the spin degrees of freedom, including stochastic terms and compare them with similar transitions from a higher excited singlet state. Within the perturbation theory the excited triplet state appears to be extremely long living, of several orders longer than the excited singlet state. The triplet state is split in magnetic field proportionally to the Zeeman energy (cf. Fig. 5). Transitions between these states numbered by $z$ components of the total spin are also hampered by the spin-type selections rules.

The rotation of the qubit spanned on the singlet and the lowest state of the triplet ones (with $S_{z}=-1$ ) can be performed by the application of varying in time magnetic field oriented parallel to the QD. The resulting Rabi-type oscillations (Appendix B) concern only the considered singlet-triplet transition which can be achieved by suitable tuning of perpendicular magnetic field (in order to avoid multi-resonances with e.g. upper triplet states split by the Pauli term).

We consider also another possibility of the state evolution (i.e. qubit rotation). Varying the perpendicular magnetic field one can shift the system to a 
singlet-triplet intersection point. In this point one can observe also the characteristic oscillations of the wave function between two degenerated states (Appendix C) due to arbitrary and even small and static perturbation (provided that $V_{01} \neq 0$ )

$$
w_{01}=2 \frac{\left|V_{01}\right|^{2}}{(\hbar \omega)^{2}}[1-\cos (\omega t)]
$$

where $w_{01}$ is the probability of transition between two degenerated states, $\hbar \omega=$ $\sqrt{\left(V_{00}-V_{11}\right)^{2}+4\left|V_{01}\right|^{2}}$, and $V_{i j}$ are matrix elements of perturbation (here, the perturbation is the longitudinal static magnetic field). By appropriate choice of $t$ it is possible to rotate the state vector in a desired manner. If to disturb then rapidly the perpendicular magnetic field the chosen state of the qubit can be achieved. In the opposite case, the case of the sufficiently slow variation of perpendicular field magnitude, the ground singlet or triplet wave function will be obtained for fields smaller or greater than the critical one, respectively (it would be employed as the reset procedure). Note that for $N \geq 4$ transitions, the possibility of simultaneous observation of the excited triplet and ground singlet states (or vice versa) [12], cf. Fig. 2a, suggests that the rapid change of the above-mentioned field can be easily achieved in experiment.

The crucial for two-qubit operations (as the "swap" operation or CNOT (i.e. controlled-NOT), cf. e.g. [28]) is the pair of interacting two-level systems. The standard CNOT operation flips target input if the control input is $|1\rangle$ and does do nothing if the control input is $|0\rangle$ (i.e. it interchanges $|10\rangle$ with $|11\rangle$ two-qubit states). To perform this procedure two separated quantum information carriers (qubits), which can interact in a controlled manner, are needed. As such system one can consider the pair of QDs. For the model let us consider the pair of He dots. This system with two electrons in each dot can be examined within the perturbation approach of Heitler-London type. The possibility of tuning, by magnetic field, the level separations in each qubit from very small to rather high allows us to enhance or diminish the role of qubits coupling (due to simple energy relations). The matrix elements of inter-dot interaction (qubits coupling) can be treated as small or big only by comparison with intra-qubit energy level separation (as we confined the system to only a pair of qubits). In the case when this separation is much greater than the energy of inter-qubit interaction, the role of this interaction is practically negligible and therefore the reconstruction of separate qubits states to entangled state does not occur. In the opposite case when the energy levels in qubits are located in the distance of the same order as the interaction, the four-electron system should be considered instead of two independent two-electron subsystems. In this case the interaction between qubits leads to entanglement of them, quite natural for the description of the entire four-particle system.

Therefore by tuning the qubit level separation via variation of the perpendicular magnetic field near the singlet-triplet transition point one can achieve the controlled, time dependent inter-qubit interaction. It seems that this scenario of two-qubit operations could be applied also to other concepts of qubit with a tunable level separation.

In the case of DiVincenzo [1, 2] proposal of spin qubit on a single electron QD, the Zeeman splitting, being the qubit level separation in that case, can be made small or big in comparison with inter-dot interaction, via tuning the magnetic field 
and therefore can allow or preserve the creation of entanglement of qubits states. It actually makes the exchange interaction (the difference between the entangled triplet and singlet states for a pair of single-spin qubits) negligible or not. In other words, two magnetic field dependent two-particle levels, singlet and triplet, intersect at a certain field (similarly as for He QD, even though electrons are located in distinct single-electron dots) and at this field the qubits interaction is switched off (we suppose here a vertically stacked dot system, contrary to the original DiVincenzo proposal, because the axial symmetry allow in this case for almost a direct application of methods from $\mathrm{He} \mathrm{QD}$ ).

Taking into account this method of two-qubit operations one could perform CNOT operation via suitably tailoring the time dependent magnetic field shifts in the vicinity of singlet-triplet transition. Let us underline that it is convenient to manage with slightly distinct dots in the coupled pair (as it is actually observed in real vertically stacked systems [10]) in order to allow the addressing, by energy, the single qubit operations.

The other methods of two-qubit operations can be also considered for a couple of He QDs. In the case of two slightly distinct qubits in the pair it is possible to find the magnetic field region in which the state of the first qubit strongly affects the states of the second one allowing for conditional logic operations. The other opportunity is to try to temporarily separate electrons in opposite sides of two stacked dots by lateral electrical gating applied to both dots in the opposite directions. It would be however realistic for not small dots and when they are located rather not too close. But in this case the interaction of qubits would be insufficient to create the entangled state. Alternatively the two-qubit operations can be performed also by mapping the information from single qubits (QDs) onto photons in cavity quantum electrodynamics (QED) but an experimental realization of this idea is not as yet completed [28].

Finally let us mention the decoherence effects. As the triplet-singlet transition needs the coupling of environment perturbations with spin of electrons, the DiVincenzo arguments related to the smaller decoherence rate in this case generally hold [2]. In the case of singlet and triplet states we have however the coupling between the orbital and spin structure in wave functions for these states. Therefore we cannot consider the separate evolution of only spin structure of these functions - such an evolution causes also a change of their orbital parts. The decoherence via orbital parts of wave functions is highly inconvenient. These problems need however separate considerations.

\section{Acknowledgment}

Supported by EU Research and Technological Development Project "Semiconductor-Based Implementation of Quantum Information Devices". 


\section{APPENDIX A}

\section{Singlet-triplet transition in $\mathrm{He}$ QD}

The singlet-triplet transition for He QD has been analysed numerically in [17]. It is however interesting to give approximate analytical formulae for crossing states which highly simplifies further applications. The He dot is described by the Hamiltonian

$$
H=\sum_{i=1}^{2}\left[-\frac{\hbar^{2}}{2 m^{*}} \Delta_{i}+\frac{1}{2} m^{*} \omega^{2} r_{i}^{2}+\frac{\hbar \omega_{c}}{2} \hat{l}_{i z}+g \hbar \omega_{c} \hat{s}_{i z}\right]+\frac{e^{2}}{\epsilon \rho},
$$

where $\omega^{2}=\omega_{0}^{2}+\frac{1}{4} \omega_{c}^{2}, \omega_{c}=e B / m^{*}, \rho=\left|r_{1}-r_{2}\right|, g$ - giromagnetic factor.

In the centre of mass and relative coordinates the wave functions corresponding to above Hamiltonian have the following form:

$$
\psi_{N M}(\boldsymbol{R}) \phi_{n m}^{\mathrm{s}, \mathrm{t}}(\rho) \chi_{S, S_{z}}^{\mathrm{s}, \mathrm{t}}\left(\sigma_{1}, \sigma_{2}\right)
$$

where $\mathrm{s}$ and $\mathrm{t}$ indicate singlet and triplet, respectively, and

$$
\begin{aligned}
& \chi_{S=0, S_{z}=0}^{\mathrm{s}}=\frac{1}{\sqrt{2}}\left[\chi_{1 / 2}\left(\sigma_{1}\right) \chi_{-1 / 2}\left(\sigma_{2}\right)-\chi_{1 / 2}\left(\sigma_{2}\right) \chi_{-1 / 2}\left(\sigma_{1}\right)\right], \\
& \chi_{S=1, S_{z}=1}^{\mathrm{t}}=\chi_{1 / 2}\left(\sigma_{1}\right) \chi_{1 / 2}\left(\sigma_{2}\right), \\
& \chi_{S=1, S_{z}=0}^{\mathrm{t}}=\frac{1}{\sqrt{2}}\left[\chi_{1 / 2}\left(\sigma_{1}\right) \chi_{-1 / 2}\left(\sigma_{2}\right)+\chi_{1 / 2}\left(\sigma_{2}\right) \chi_{-1 / 2}\left(\sigma_{1}\right)\right], \\
& \chi_{S=1, S_{z}=-1}^{\mathrm{t}}=\chi_{-1 / 2}\left(\sigma_{1}\right) \chi_{-1 / 2}\left(\sigma_{2}\right),
\end{aligned}
$$

and

$$
\psi_{N M}=\frac{1}{L} G_{N|M|}(R / L) \frac{1}{\sqrt{2 \pi}} \exp \left(\mathrm{i} M \theta_{R}\right)
$$

where $L=\sqrt{\hbar / M_{0} \omega}, M_{0}=2 m^{*}$, and

$$
\begin{aligned}
& \phi_{n m}^{\mathrm{s}}(\boldsymbol{\rho})=\phi_{n m}^{\mathrm{s}}(-\boldsymbol{\rho})=\frac{1}{l} g_{n|m|}(\rho / l) \frac{1}{\sqrt{2 \pi}} \exp \left(\mathrm{i} m \Theta_{\rho}\right), \quad m=2 k, \\
& \phi_{n m}^{\mathrm{t}}(\boldsymbol{\rho})=-\phi_{n m}^{\mathrm{t}}(-\boldsymbol{\rho})=\frac{1}{l} g_{n|m|}(\rho / l) \frac{1}{\sqrt{2 \pi}} \exp \left(\mathrm{i} m \Theta_{\rho}\right), \quad m=2 k+1,
\end{aligned}
$$

where $l=\sqrt{\hbar / m_{0} \omega}, m_{0}=m^{*} / 2, k$ - integer.

The corresponding eigenenergies have the following form:

$\epsilon_{N M, n m, S S_{z}}=\epsilon_{N M}+\epsilon_{n m}+g \hbar \omega_{c} S_{z}$,

where $\epsilon_{N M}=\hbar \omega(N+1)+M \hbar \omega_{c} / 2$ and $\epsilon_{n m}=\hbar \omega E_{n|m|}+m \hbar \omega_{c} / 2$. 
Hence for the first pair of intersecting levels, denoted by $|0\rangle$ and $|1\rangle$, we have

$$
|0\rangle=\psi_{00} \phi_{00} \chi_{00}
$$

where

$$
\begin{aligned}
& \psi_{00}=\frac{1}{L \sqrt{\pi}} \exp \left(-R^{2} / 2 L^{2}\right), \\
& \phi_{00}=\frac{1}{l \sqrt{2 \pi}} A_{0} \exp \left[-\frac{1+b_{0}}{2}(\rho / l)^{2}+2 \rho / a\right],
\end{aligned}
$$

with $a=\epsilon \hbar^{2} / m_{0} e^{2}$,

$$
A_{0}^{2}=\frac{2\left(1+b_{0}\right)}{\frac{4 l / a}{\sqrt{1+b_{0}}} \exp \left[\frac{(2 l / a)^{2}}{1+b_{0}} \frac{\sqrt{\pi}}{2}\left(1+\Phi\left(\frac{2 l / a}{\sqrt{1+b_{0}}}\right)\right)\right]+1}
$$

here $\Phi$ is the error function, and

$$
|1\rangle=\psi_{00} \phi_{1-1} \chi_{1-1}
$$

where

$$
\begin{gathered}
\phi_{1-1}=\frac{\rho}{l^{2} \sqrt{\pi}} A_{1} \exp \left[-\frac{1+b_{1}}{2}(\rho / l)^{2}+2 \rho / 3 a\right] \exp \left(-\mathrm{i} \Theta_{\rho}\right), \\
A_{1}^{2}=\frac{8\left(1+b_{1}\right)^{2}}{\frac{8 l / 3 a}{\sqrt{1+b_{1}}}\left[3+\frac{2(2 l / 3 a)^{2}}{1+b_{1}}\right] \exp \left[\frac{(2 l / 3 a)^{2}}{1+b_{1}} \frac{\sqrt{\pi}}{2}\left(1+\Phi\left(\frac{2 l / 3 a}{\sqrt{1+b_{1}}}\right)\right)\right]+4 \frac{(2 l / 3 a)^{2}}{1+b_{1}}+4} .
\end{gathered}
$$
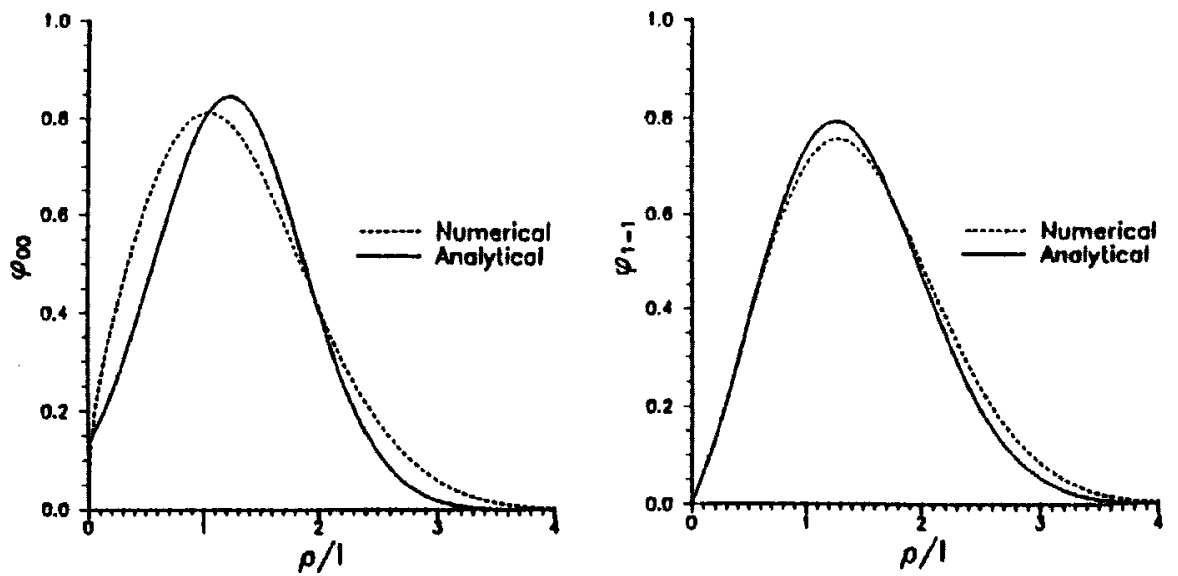

Fig. 6. Comparison of analytical approach with numerical calculations for singlet (left) and triplet (right) wave functions in He dot. 
The corresponding eigenenergies are

$$
\epsilon_{00,00,00}=\hbar \omega\left(\frac{E_{00}}{2}+1\right)
$$

and

$$
\epsilon_{00,1-1,1-1}=\hbar \omega\left(\frac{E_{11}}{2}+1\right)-\frac{\hbar \omega_{c}}{2}(1+2 g) .
$$

In the above formulae the parameters $b_{0}$ and $b_{1}$ are treated as variational parameters which allow for evaluation of $E_{00}$ and $E_{1-1}$ with respect to magnetic field. For various QD confinements given by $\hbar \omega_{0}$ we determine the magnetic field for the first singlet-triplet transition. For instance, for GaAs QD with $\hbar \omega_{0}=$ $17.4 \mathrm{meV}, B^{*}=12 \mathrm{~T}$, for $\hbar \omega_{0}=5.2 \mathrm{meV}, B^{*}=3.2 \mathrm{~T}$ and for $\hbar \omega_{0}=2.5 \mathrm{meV}$, $B^{*}=1 \mathrm{~T}$. These values correspond well with numerical results from [17].

The accuracy of this analytical approach is checked also by direct comparison with numerical calculations, cf. Fig. 6.

\section{APPENDIX B}

\section{Rabi-type oscillations for singlet-triplet He QD qubit}

The rotation of the qubit defined by two levels - the singlet ground state of He QD at a field in the left vicinity of the critical field and the excited triplet state with $S_{z}=-1$ at the same field - can be performed by Rabi-type oscillations. These oscillations are the response to an external longitudinal dynamical magnetic field which we assume in the following form:

$$
\boldsymbol{B}(r, t)=\boldsymbol{B}(\boldsymbol{r}) \exp (-\mathrm{i} \omega t)+\text { h.c. }
$$

where $\boldsymbol{B}(\boldsymbol{r})=(B \exp (\mathrm{i} \boldsymbol{k} \cdot \boldsymbol{r}), 0,0) \simeq(B+\boldsymbol{b} \cdot \boldsymbol{r}, 0,0), \boldsymbol{b}=\mathrm{i} \boldsymbol{k} B$.

This field leads to the perturbation in Hamiltonian for He QD

$w=w_{0} \exp (-\mathrm{i} \omega t)+w_{0}^{+} \exp (\mathrm{i} \omega t)$

and $w_{0}=g e \hbar / m^{*}\left[\left(\hat{s}_{1 x}+\hat{s}_{2 x}\right)(B+\boldsymbol{b} \cdot \boldsymbol{R})+\left(\hat{s}_{1 x}-\hat{s}_{2 x}\right) \boldsymbol{b} \cdot \boldsymbol{\rho} / 2\right]$.

For $|0\rangle$ and $|1\rangle$ states described in Appendix $\mathrm{A}\langle 0|w| 0\rangle=\langle 1|w| 1\rangle=0$, and

$$
\langle 1|w| 0\rangle=w_{10} \exp \left[\mathrm{it}\left(\frac{E_{1}-E_{0}}{\hbar}-\omega\right)\right]+w_{01}^{*} \exp \left[\mathrm{i} t\left(\frac{E_{1}-E_{0}}{\hbar}+\omega\right)\right] \text {, }
$$

where $w_{10}=\left(g e \hbar / \sqrt{2} m^{*}\right)\left(b_{x} \gamma l / 2-\mathrm{i} b_{y} \gamma l / 2\right)$, and $\gamma=\int_{0}^{\infty} x^{2} g_{11}(x) g_{00}(x) \mathrm{d} x$.

If we identify the qubit with the state $c_{0}|0\rangle+c_{1}|1\rangle$ then

$$
\begin{aligned}
& \mathrm{i} \hbar \frac{\mathrm{d} c_{1}}{\mathrm{~d} t}=w_{10} \exp (\mathrm{i} \epsilon t) c_{0}, \\
& \mathrm{i} \hbar \frac{\mathrm{d} c_{0}}{\mathrm{~d} t}=w_{10}^{*} \exp (-\mathrm{i} \epsilon t) c_{1},
\end{aligned}
$$

where $\epsilon=\left(E_{1}-E_{0}\right) / \hbar-\omega$.

The solution of the above equation has the following form:

$u(t)=A \exp [\mathrm{i}(\epsilon / 2 \pm \Omega) t]$,

where $u=c_{0} \exp (\mathrm{i} \epsilon t)$ and $\Omega=\sqrt{\epsilon^{2} / 4+\left|w_{10}\right|^{2} / \hbar^{2}}$. 
In resonance, when $\epsilon=0$, we observe Rabi-type oscillations

$$
\begin{aligned}
& |0\rangle(t)=|0\rangle \cos (\Omega t) \exp \left(-\mathrm{i} E_{0} t / \hbar\right)-|1\rangle \mathrm{i} w_{10}^{*} /\left|w_{10}\right| \sin (\Omega t) \exp \left(-\mathrm{i} E_{1} t / \hbar\right) \\
& |1\rangle(t)=|0\rangle \mathrm{i} w_{10} /\left|w_{10}\right| \sin (\Omega t) \exp \left(-\mathrm{i} E_{0} t / \hbar\right)+|1\rangle \cos (\Omega t) \exp \left(-\mathrm{i} E_{1} t / \hbar\right)
\end{aligned}
$$

From the above formulae it follows that the probability of transition between two states of our subspace is proportional to $\sin ^{2}(\Omega t)=[1-\cos (\Omega t / 2)] / 2$.

\section{APPENDIX C}

\section{Rotation of the singlet-triplet qubit by shifts of perpendicular magnetic field}

While shifting the perpendicular magnetic field in the direction of the intersection point we transform the qubit without loosing information. When we reach however the intersection point the new opportunity appears. In this point two states described in Appendix A span the two-dimensional subspace corresponding to the same energy value - it is a degeneracy point. In the case of the two-level degenerated system one observes the time-dependent rotation of the state (within the two-dimensional subspace). This rotation is induced by any static perturbation $V$ for which the matrix element $\langle 0|V| 1\rangle$ is nonzero. According to perturbation theory for degenerated levels one can determine the corrections to the energy (which remove the degeneracy), cf. [29]

$$
\Delta E=\left(V_{00}+V_{11} \pm \hbar \omega\right) / 2
$$

and

$$
\hbar \omega=\sqrt{\left(V_{00}+V_{11}\right)^{2}+4\left|V_{01}\right|^{2}} .
$$

These energy corrections lead to the rotation of the state, which can be described by the transition probability

$$
w_{01}=2 \frac{\left|V_{01}\right|^{2}}{(\hbar \omega)^{2}}[1-\cos (\omega t)]
$$

For small perturbations the period of transition is sufficiently long.

In the chosen moment of time one can rapidly remove the system from the intersection point by the change of perpendicular magnetic field. In that manner it is possible to map the quantum state prepared in the degeneracy point into the qubit state with different energy levels. For a longer time (and it depends of the choice of qubit location in the vicinity of the singlet-triplet transition point) the qubit eventually will transform to the ground state (singlet or triplet for left and right position with respect to the intersection point). This last procedure could be treated as the reset scheme for a singlet-triplet QD qubit. 


\section{APPENDIX D}

\section{The vertically stacked He QDs}

The Hamiltonian for this system is given by

$$
H=H_{0}\left(\boldsymbol{r}_{1}, \boldsymbol{r}_{2}\right)+H_{0}\left(\boldsymbol{r}_{1}^{\prime}, \boldsymbol{r}_{2}^{\prime}\right)+w\left(\boldsymbol{r}_{1}, \boldsymbol{r}_{2}, \boldsymbol{r}_{1}^{\prime}, \boldsymbol{r}_{2}^{\prime}\right)
$$

where $H_{0}$ is defined for a single He QD in Appendix A. The interaction term

$$
\begin{gathered}
w\left(\boldsymbol{r}_{1}, \boldsymbol{r}_{2}, \boldsymbol{r}_{1}^{\prime}, \boldsymbol{r}_{2}^{\prime}\right)=\frac{e^{2}}{\epsilon \sqrt{\left(\boldsymbol{r}_{1}-\boldsymbol{r}_{1}^{\prime}\right)^{2}+x^{2}}}+\frac{e^{2}}{\epsilon \sqrt{\left(\boldsymbol{r}_{1}-\boldsymbol{r}_{2}^{\prime}\right)^{2}+x^{2}}} \\
+\frac{e^{2}}{\epsilon \sqrt{\left(\boldsymbol{r}_{2}-\boldsymbol{r}_{1}^{\prime}\right)^{2}+x^{2}}}+\frac{e^{2}}{\epsilon \sqrt{\left(\boldsymbol{r}_{2}-\boldsymbol{r}_{2}^{\prime}\right)^{2}+x^{2}}},
\end{gathered}
$$

where $x$ is the vertical separation between dots. The perturbation with respect to $w\left(\boldsymbol{r}_{1}, \boldsymbol{r}_{2}, \boldsymbol{r}_{1}^{\prime}, \boldsymbol{r}_{2}^{\prime}\right)$ reproduces the Heitler-London approach for molecules. As the zeroth approximation we take states of two separated dots truncating the corresponding Hilbert space to only two lowest states (singlet and triplet ones). The perturbation is simple if we take only the $S_{z}=-1$ triplet state. When, however, the levels separations in QDs are of the similar order as the interaction $w$ then the perturbation methods cannot be applied. In this case we deal actually with strongly interacting four-electron system. In the case when two coupled dots are of a distinct diameter (which allow for a single qubit addressing by energy) by tuning magnetic field we can achieve the various interlevel energy distances and it is possible to affect levels significantly by the interaction $w$. In particular it is possible to arrange the conditional separation of levels in one dot with respect to the state occupation in the second dot. Note that if the Zeeman splitting is of the order of interlevel distances, then three components of triplet state have to be taken into account (then the state of four electrons in the system can be numbered by the total spin $S=0,1,2$ with corresponding $S_{z}$ components split by Pauli term).

\section{References}

[1] A. Imamoglu, D.D. Awschalom, G. Burkard, D. DiVincenzo, D. Loss, M. Sherwin, A. Small, Phys. Rev. Lett. 83, 4204 (1999).

[2] D. Loss, D. DiVincenzo, Phys. Rev. A 57, 120 (1998).

[3] P. Zanardi, F. Rossi, Phys. Rev. B 59, 6170 (1999).

[4] P. Zanardi, F. Rossi, Phys. Rev. Lett. 81, 4752 (1998).

[5] N.H. Bonadeo, J. Erland, D. Gammon, D.S. Katzer, D. Park, D.G. Steel, Science 282, 1473 (1998).

[6] A.P. Heberle, J.J. Baumberg, K. Kohler, Phys. Rev. Lett. 75, 2598 (1995).

[7] J.M. Kikkawa, D.D. Awschalom, Phys. Rev. Lett. 80, 4213 (1998).

[8] D. Bouwmeester, A. Ekert, A. Zeilinger, The Physics of Quantum Information, Springer-Verlag, Berlin 2000.

[9] J. Preskill, Quantum Information and Computation, http://www.theory.caltech.edu/ preskill/ph229 (1998). 
[10] T. Lundstrom, W. Schoenfeld, H. Lee, P.M. Petroff, Science 286, 2312 (1999).

[11] S. Fafard, Physica E 8, 107 (2000).

[12] S. Tarucha, D.G. Austing, Y. Tokura, W.G. van der Wiel, L.P. Kouwenhoven, Phys. Rev. Lett. 84, 2485 (2000).

[13] S. Tarucha, D.G. Austing, T. Honda, R.J. van der Hage, L.P. Kouwenhoven, Phys. Rev. Lett. 77, 3613 (1996).

[14] L.P. Kouwehoven, T.H. Oosterkamp, M.W.S. Danoesastro, M. Eto, D.G. Austing, T. Honda, S. Tarucha, Science 278, 1788 (1997).

[15] L. Jacak, P. Hawrylak, A. Wójs, Quantum Dots, Springer Verlag, Berlin 1998.

[16] M. Rontani, F. Rossi, F. Manghi, E. Molinari, Phys. Rev. B 59, 10165 (1999).

[17] M. Wagner, U. Merkt, A. Chaplik, Phys. Rev. B 45, 1951 (1992).

[18] G. Goldoni, F. Rossi, E. Molinari, Physica B 272, 518 (1999).

[19] U. Hohenester, F. Rossi, E. Molinari, Solid State Commun. 111, 187 (1999).

[20] U. Hohenester, F. Rossi, E. Molinari, Physica B 272, 1 (1999).

[21] S. Raymond, S. Fafard, P.J. Poole, A. Wojs, P. Hawrylak, S. Charbonneau, D. Leonard, R. Leon, P.M. Petroff, J.L. Merz, Phys. Rev. B 54, 11548 (1996).

[22] M. Rontani, F. Rossi, F. Manghi, E. Molinari, Solid State Commun. 112, 151 (1999).

[23] A. Wojs, P. Hawrylak, Solid State Commun. 100, 487 (1996).

[24] A. Wójs, P. Hawrylak, L. Jacak, Acta Phys. Pol. A 90, 1108 (1996).

[25] S. Hameau, Y. Guldner, O. Verzelen, R. Ferreira, G. Bastard, J. Zeman, A. Lemaitre, J.M. Gerard, Phys, Rev. Lett. 83, 4152 (1999).

[26] A. Wojs, P. Hawrylak, Phys. Rev. B 53, 10841 (1996).

[27] M. Rontani, F. Rossi, F. Manghi, E. Molinari, Appl. Phys. Lett. 72, 957 (1998).

[28] Ch. Bennet, D. DiVincenzo, Nature 404, 247 (2000).

[29] L. Landau, E. Lifšic, Quantum Mechanics, PWN, Warsaw 1979 (in Polish).

[30] A. Wojs, P. Hawrylak, S. Fafard, L. Jacak, Phys. Rev. B 54, 5604 (1996). 